\title{
Opnet Based Performance Analysis and Comparison Among Different Physical Network Topologies
}

\author{
Noor Najeeb Qaqos¹, Subhi R. M. Zeebaree², Bzar Khidir Hussan³ \\ ${ }^{1}$ Shekhan Technical Institute, Duhok Polytechnic University, Duhok, Kurdistan Region - Iraq \\ ${ }^{2}$ College of Engineering, Nawroz University, Duhok, Kurdistan Region - Iraq \\ ${ }^{3}$ Department of ISE, Engineering Technical College, Erbil Polytechnic University, Duhok, Kurdistan Region - Iraq
}

\begin{abstract}
Physical network topology is the configuration of computers, cables and other peripherals as a geometrical shape. There are many types of topologies each of them has many advantages and disadvantages. For high performance Local Area Networks (LANs), the builder of a network should select the best physical network topology. This paper analyzes three basic network topologies including bus, star and ring for different number of connected nodes (various network topology size) using OPNET simulator. Four scenarios are configured for each topology with number of connected nodes equal to 5, 10, 15 and 20 for senario1, 2, 3 and 4 respectively. In this analysis, four parameters investigated in Delay (Sec.) for the complete network, Load (Bits/Sec.), Traffic Received (Bits/Sec.) and the number of Collision for single node (server). Performance comparison is made in each topology separately based on various network size and another comparison is also made between these topologies for the same number of connected devices. The comparison results indicate that performance decreased when the network size increased and also show that bus topology is more effected than two other topologies.

KEYWORDS : OPNET Simulator, Physical Network Topology, Delay, Load, Traffic Received, Number of Collision.
\end{abstract}

\section{INTRODUCTION}

Network topology divided into two types : physical and logical topology. While physical topology is the technology for arrangement of different computer elements such as nodes, links etc., the logical topology is the way where the data passed from node to another in the network. There are many types of physical topologies each of them suited for a specific task and it has many advantages and disadvantages. The selection of a suitable topology for a designed network depends on different factors such as cost, number of nodes being used, usage application servers (FTP, HTTP and Database,......etc.) and also the required response times. There are three basic types of physical topologies (bus, star and ring). Most networking software simulators used to create, simulate and support all topologies. Performance

Academic Journal of Nawroz University

(AJNU) Volume 7, No 3 (2018).

Received 22 Feb 2018;

Regular research paper : Published 20 June 2018

Corresponding author's e-mail : noor_msc86@yahoo.com

Copyright (C2018 Noor Najeeb Qaqos.

This is an open access article distributed under the

Creative Commons Attribution License. analysis of any network topology should be done using software simulators before practical design of network. Simulation, modeling and analysis for different parameters of ring topology for computer applications in network having 10 Ethernet workstations using OPNET Simulator proposed by Harmeet, et al. [1] and they concluded that the throughput of the topology decreased when the network size increased. Bobbinpreet [2] simulated and analyzed star topology in LANs using OPNET simulator for different network size and various duration time of simulation. Study and analysis of various kinds of network topology for data communication are considered without using any software simulation and the advantages and disadvantages are described by Santanu and Pinaki [3]. Presentation and a comparative analysis of different topologies based on network-on-chip architectures introduced by Mridula, et al. [4]. Sushruta, et al. [5] presented an asuccinct study of different types of topology and also a study on various types of devices with its functionalities that used with each types of these topologies. A study and comparison between the performance of tree and ring topologies in distributed systems achieved by Huang and Bode [6] and concluded from their study that tree network topology is superior to the ring network when the size of the system and the 
data transmitted in the system have large payloads. Pankaj and Dilpreet [7] analyzed the performance of ring topology based on token ring method for both balanced and unbalanced load and they indicated that the total delay encountered by media access and packet size for balanced load is lower than unbalanced load for real time applications. This paper is organized as follows : Besides this introductory section, Section 2 describes the basic three types of physical network topology used in this work. Section 3 introduces to OPNET Software that used as a simulation tool, a configuration and implementation of network topologies using OPNET simulator are described in section 4. Performance analysis and result comparisons are presented in section 5. Finally, conclusions are drawn based on my results.

\section{PHYSICAL NETWORK TOPOLOGIES}

Physical network topology means the arrangement of devices such as workstations, remote terminals and servers on a computer network through the actual links (cables) that transmit and receive data [3]. It refers to how these hardware devices and cables physically connected. There are many basic types of topologies, in this work three basic types (bus, star and ring) are described.

\section{* BUS Topology}

It consists of a main cable with a terminator at each ends where all nodes are connected to this cable, as shown in figure(1a). When a node wants to send a message, the bus topology carries the message along the main cable. As the message arrives at each node (device), the destination address part contained in the message checks by the node to show if it matches with its address or not, if the address matched the node received the message else the node does not receive it. Coaxial cables usually used to connect such type of topology.

\section{* STAR Topology}

It is the most common type of topologies and consists of a central unit where each node in the network connected to it. The central unit may be hub, switch or concentrator, as shown in figure (1b). When a node sends data to another node, the data passed through a central unit and then to received node. Twisted pair cable is the most common type to connected this type of topology.

\section{* RING Topology}

In this type of topology each node connected to two other nodes where all nodes are connected to one another as a circle, as shown in figure (1c). Data are transmitted in one direction (clockwise or counterclockwise) around the ring. Each node accepts and response to the data that addressed to it and forwards the other data that not addressed to it to other nodes in the ring. The nodes in ring topology act as a repeater to send data to other nodes in the ring. The type of cable used in ring networks is twisted pair.

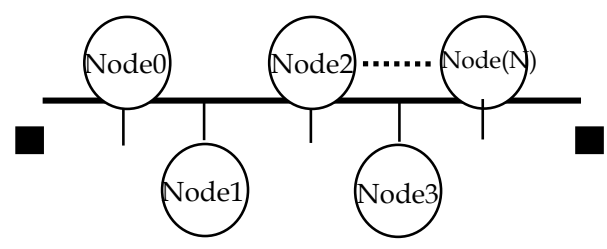

(a) Bus

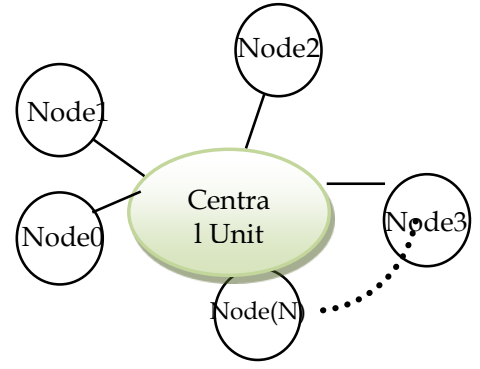

(b) Star

Fig (1) : Physical Network Topologies

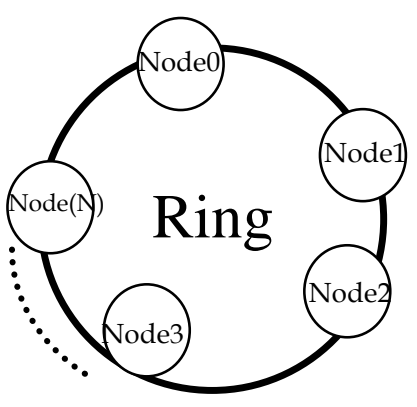

(c) Ring

\section{SIMULATION TOOL}

OPNET (Optimized Network Engineering Tool) simulator can be used to simulate any types of network [8], so this tool is used in this paper to simulate and analyze the three types of network topology models (bus, star and ring topology). For performance analysis and comparison two types of parameters can be selected : global parameters (that is used to analyze the complete network) and local parameters (used to analyze each node connected in the network separately). Network topology model can be created using three methods. Firstly, you can import the topology. Secondly, you can drag network objects by mouse from the active palette into the displayed workspace and finally by using rapid configuration. The best and convenient method is to use rapid configuration. This method is very simple and the network created by selecting the type of topology, number and types of connected nodes and also the type of links that used to transfer data in the network. In this paper, three physical network topologies are created using rapid configuration method. Each type of network topology is client.server network and has the same components and characteristics (workstations, FTP server with file size $=10$ Megabytes and link to transfer data between devices with data rate equal to 10 
Megabytes/sec.). The only different thing among these topologies is the configuration of nodes in a network (different physical topologies).

\section{Configuration of Bus, Star and Ring Topologies}

Each topology configured using four scenarios. All the workstations and server in each scenario are connected through cables with data rate transfer equal to 10 Megabytes per second. The cable type used in bus topology is coaxial cable while in star and ring is twisted pair cable. The only difference among these four scenarios is the number of devices in the network where the number equal to 5,10,15 and 20 for scenario 1,2,3 and 4 respectively. Figures (2,3 and 4$)$ show the four scenarios for each topology using rapid configuration method. Performance of these three basic topologies analyzed using two types of parameters : Global parameter for complete network (Delay (sec.)) and three local parameters for single node set respect to server node in the network (Load (bits/sec.), traffic received (bits/sec.) and number of collision). It can be describe the meaning of these parameters as :.

1. Delay (sec.) : Delay is a time difference in sending a single unit of information such as bit, byte, frame, and packet from source to destination.

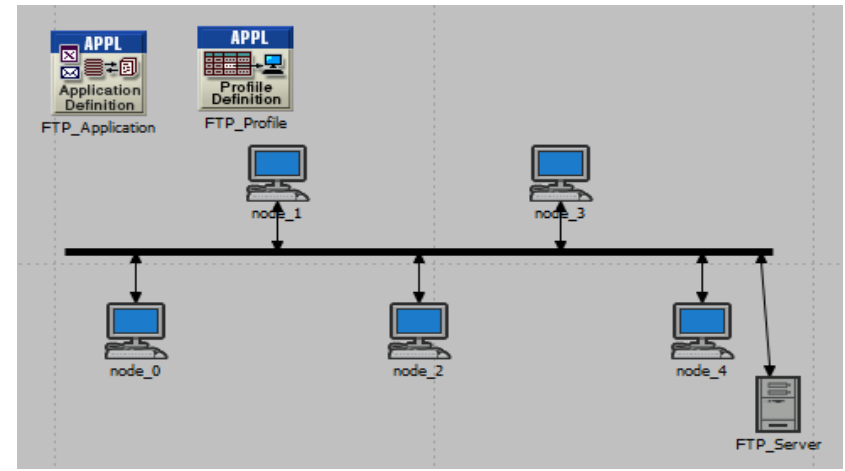

(a) 5 devices with FTP server

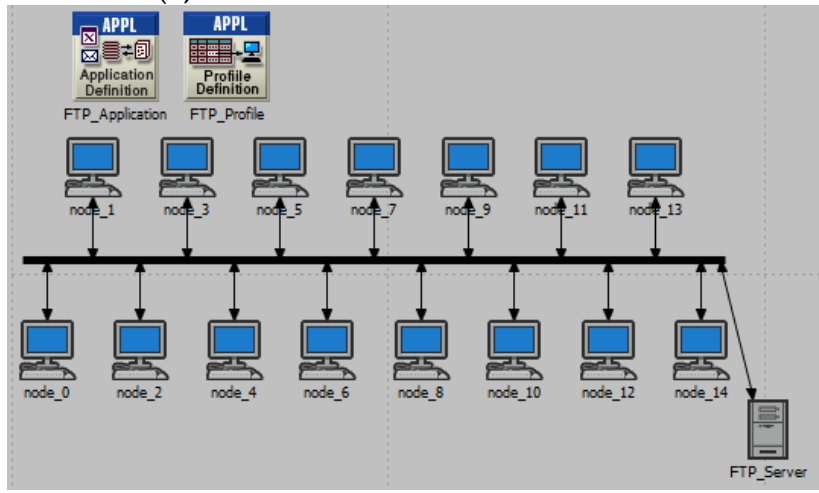

(c) 15 devices with FTP server
2. Load (bits/sec) or (packets/sec) : Load (in bits/sec or in packets/sec) sent to ethernet layer from all other higher layers in specific node (such as a server node in this work).

3. Traffic Received (bits/sec) or (packets/sec) : Throughput (in bits/sec or in packet/sec) of the data forwarded by the ethernet layer to the higher layers in a specific node (as an example of server node in this work).

4. Collision Account : the total number of collisions encountered by specific node (a server in this work) during the transmissions of packets in the network.

The simulation of four scenarios for bus, star and ring topology is shown in figures (2, 3 and 4), respectively. After that simulate the system, set all parameters and adjust the duration of time to 1 hour which means that the results are for a network system operated for 1 hour only. Then run the project and view the result graph for selected parameters.

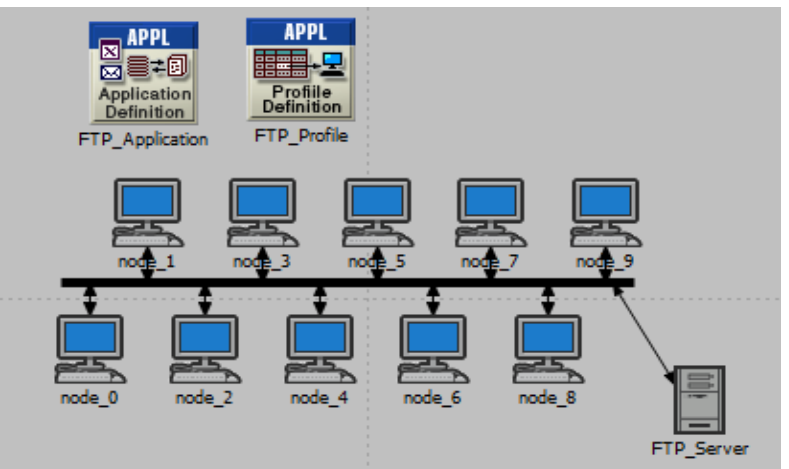

(b) 10 devices with FTP server

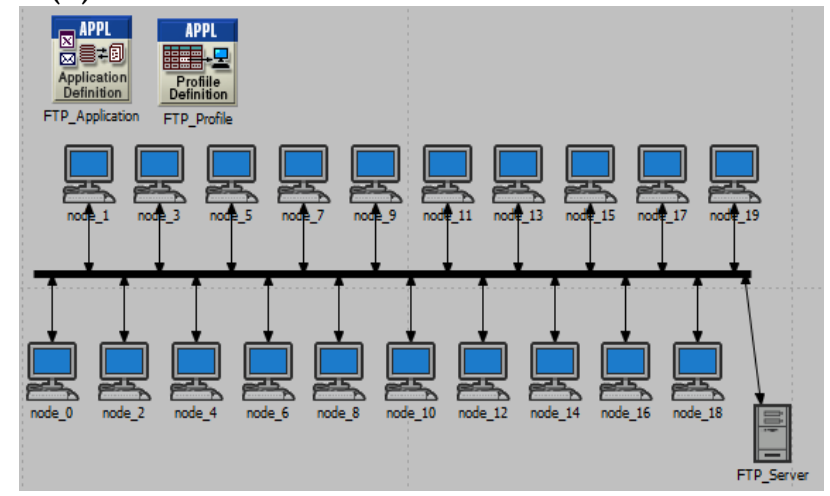

(d) 20 devices with FTP server

Fig. (2) Four Scenarios Bus Topology 


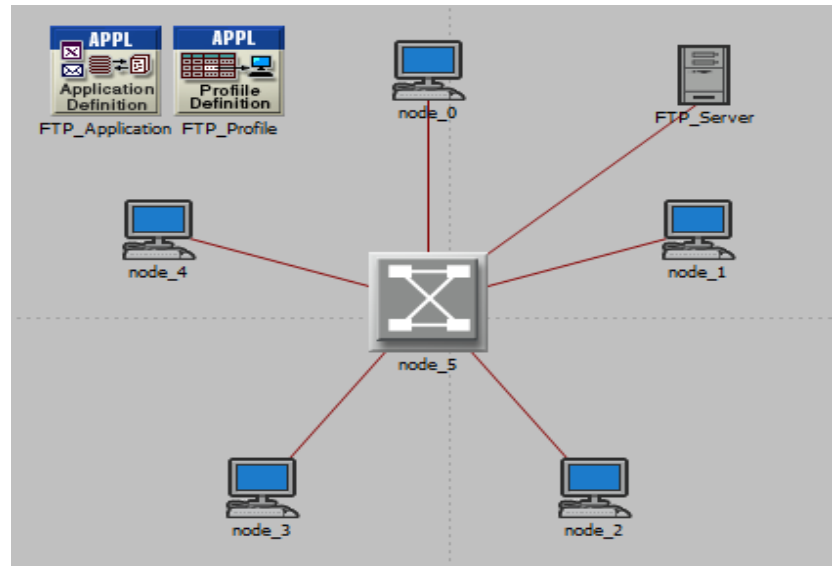

(a) 5 devices with FTP server

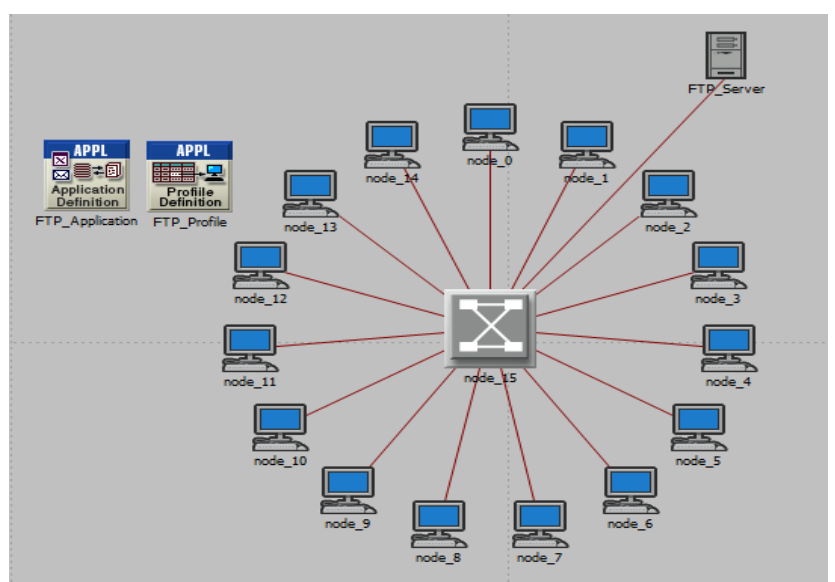

(c) 15 devices with FTP server

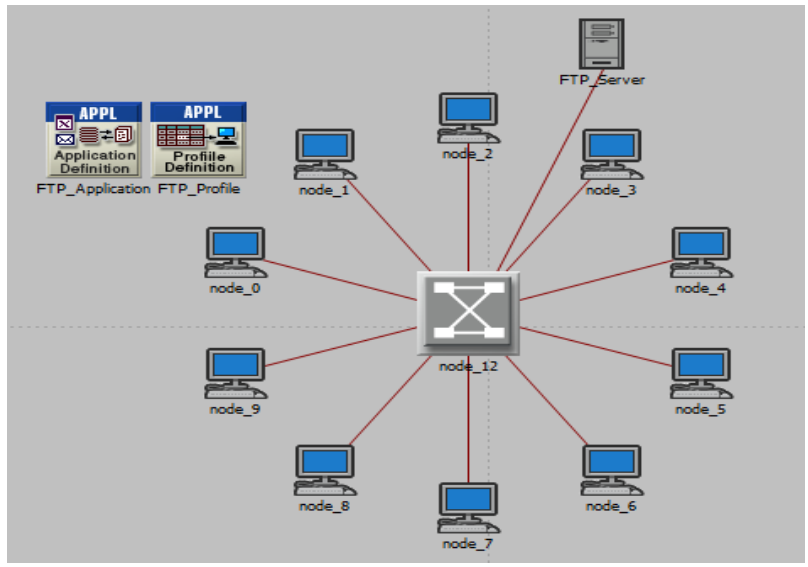

(b) 10 devices with FTP server

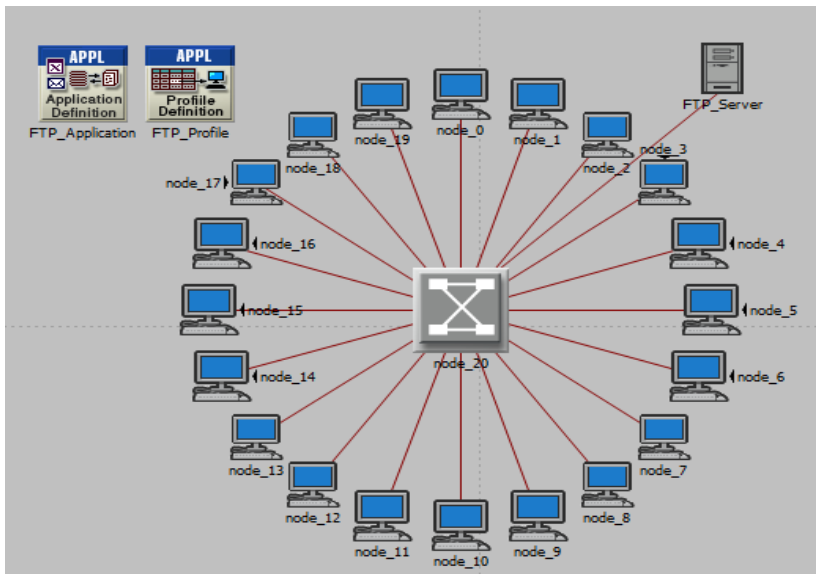

(d) 20 devices with FTP server

Fig. (3) Four Scenarios Star Topology

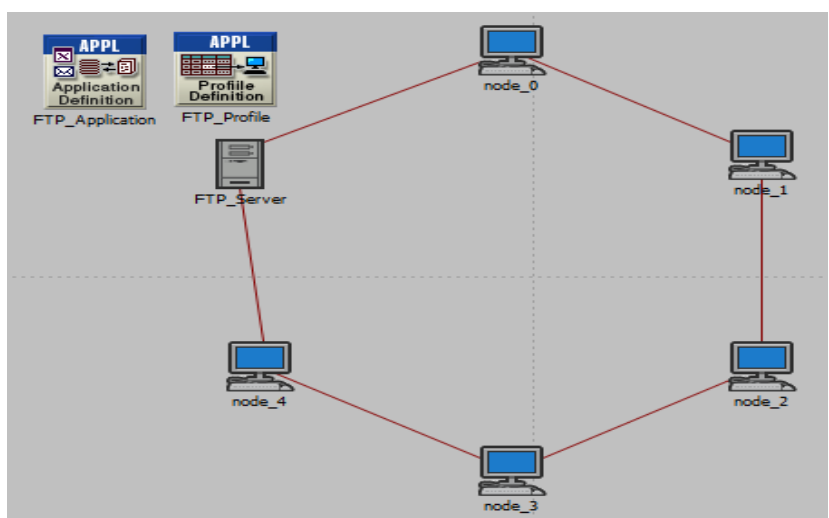

(a) 5 devices with FTP server

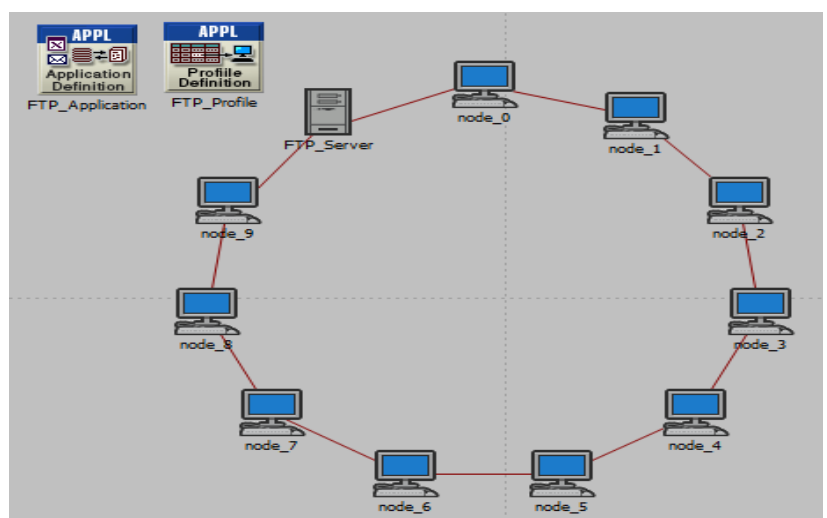

(b) 10 devices with FTP server 


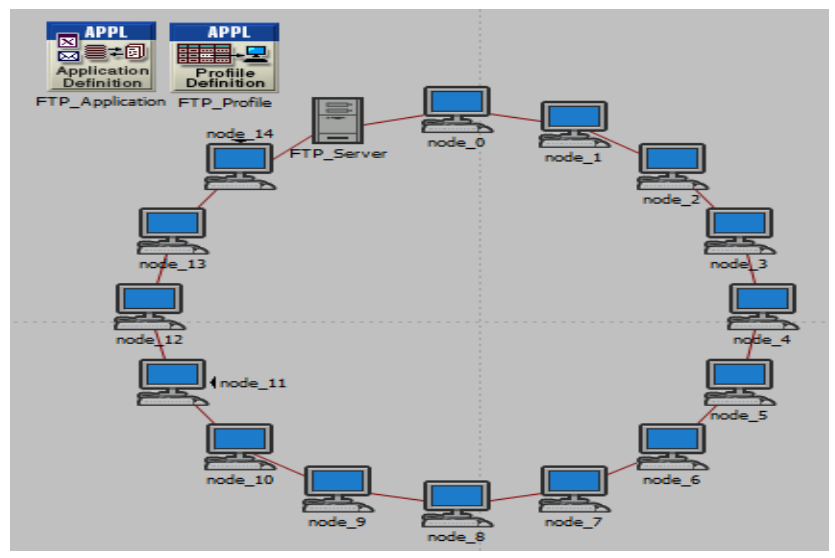

(c) 15 devices with FTP server

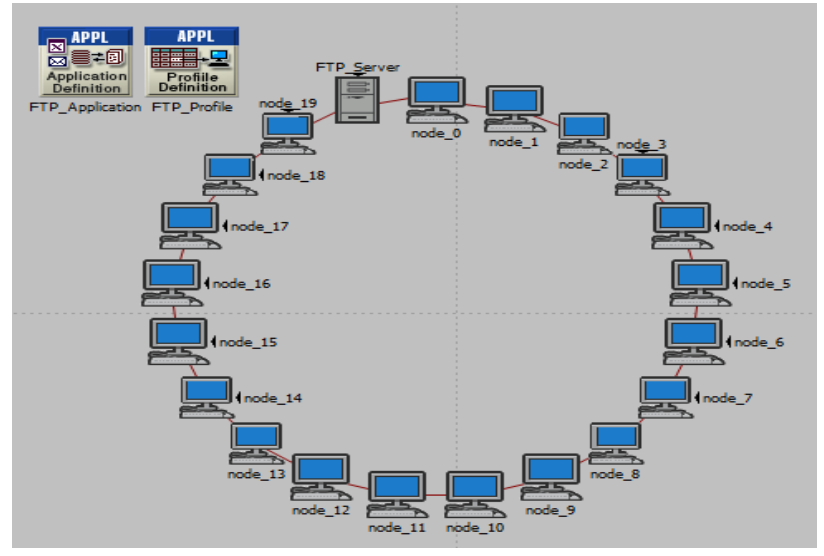

(d) 20 devices with FTP server

Fig. (4) Four Scenarios Ring Topology

\section{RESULTS AND PERFORMANCE ANALYSIS}

When the four scenarios for each topology are designed and configured, the global parameter (Delay (sec.) for complete network) is selected and the three local parameters (Load (Bits/Sec.), number of Collision and Traffic Received (Bits/Sec.)) are selected also for server node only. The delay parameter is performed and a comparison is made among four scenarios for each topology as a graphical form as shown in figures $(5,6$ and 7). It can be seen from figure (6) that the delay in star topology is directly proportional to the number of nodes connected to the network. Whereas increasing or decreasing delay in bus and ring topologies doesn't depend on the number of nodes connected in the network which means that a delay changed randomly with different number of connected nodes as shown in figures (5 and 7). Similarly a comparison can be made and prepared in the form of table for parameters used to measure and analysis the performance for server node. From table (1), It can be seen for all of three topologies that the load and traffic received of a server increased when the number of connected nodes increased and vice versa. Moreover, the number of collision in bus topology increased by increasing the connected nodes because in this type of topology the same bus (trunk cable) is used to transmit data through the network so more than one node can send data at the same time in the same cable. Whereas the number of collision for star and ring topologies equal to zero for different number of connected nodes in the network due to star topology does not use the same cable to transfer data and also in ring topology despite of data are transferred through the same ring cable that connected the nodes each by others but permission to transmit data is granted by a packet called token that move around the ring so a node cannot transmit data in the network when the token is full and nodes must wait until the empty of a token so that no more than one node can send data at the same time which means that no collision occurs in this type of topology. Also the table shows that there is a sudden change in the load, number of collision and traffic received for the server node of bus topology when the number of nodes become more than 15 which means that the bus topology performance is more effected than two other topologies by increasing the number of connected nodes. 


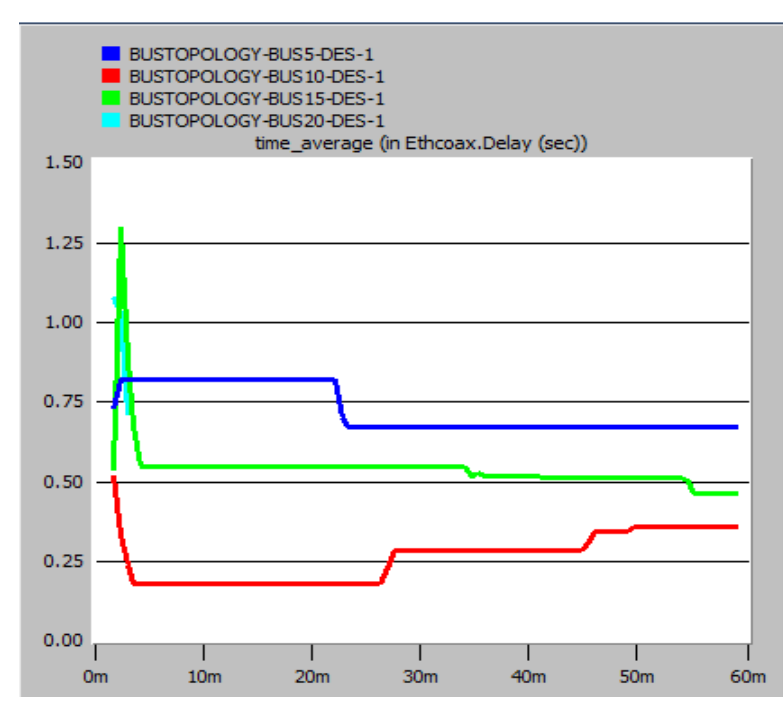

Fig. (5) Delay for Bus Topology

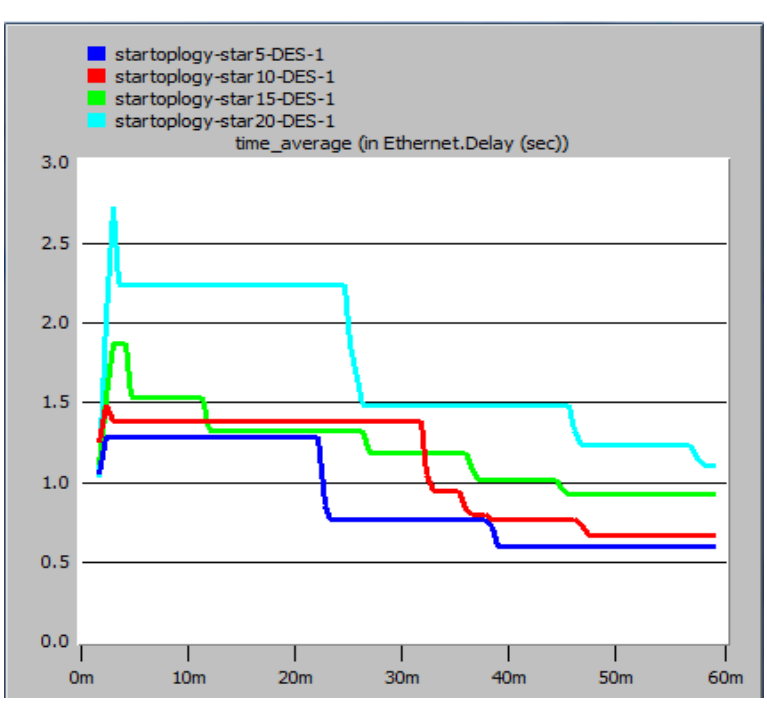

Fig. (6) Delay for Star Topology

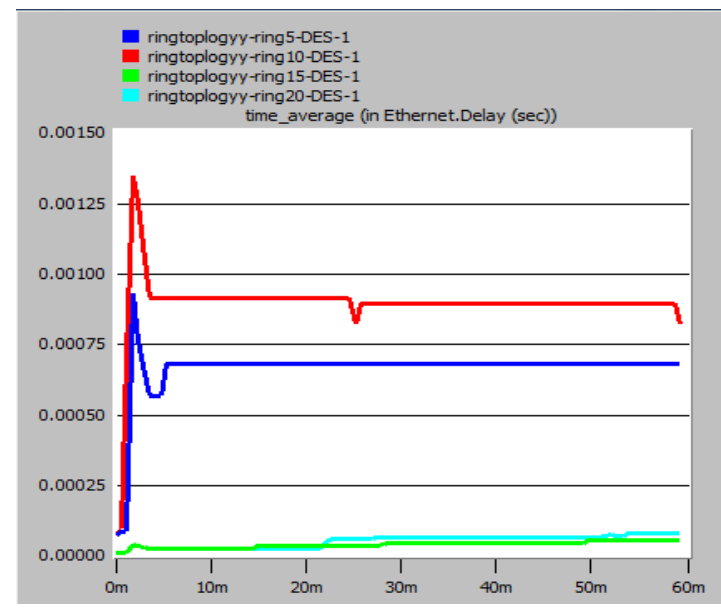

Fig. (7) Delay for Ring Topology

Table (1) : Comparison results amongst three topologies for four scenarios

\begin{tabular}{|c|c|c|c|c|}
\hline Topology Type & Scenarios & Load (bits/sec.) & $\begin{array}{c}\text { Traffic Received } \\
\text { (bits/sec.) }\end{array}$ & $\begin{array}{c}\text { Number of } \\
\text { Collision }\end{array}$ \\
\hline \multirow{4}{*}{ BUS Topology } & Scenario1 & 92886 & 47619 & 2197 \\
\cline { 2 - 5 } & Scenario2 & 96229 & 208815 & 5673 \\
\cline { 2 - 5 } & Scenario3 & 211749 & 233797 & 5992 \\
\cline { 2 - 5 } & Scenario4 & 2719555 & 1596289 & 14268 \\
\hline \multirow{4}{*}{ STAR Topology } & Scenario1 & 93,289 & 70,672 & 0 \\
\cline { 2 - 5 } & Scenario2 & 96,114 & 231,822 & 0 \\
\cline { 2 - 5 } & Scenario3 & 234,244 & 234,240 & 0 \\
\cline { 2 - 5 } RING Topology & Scenario4 & 326,741 & 258,903 & 0 \\
\hline & Scenario1 & 92653 & 68,502 & 0 \\
\cline { 2 - 5 } & Scenario2 & 115,392 & 115,392 & 0 \\
\cline { 2 - 5 } & Scenario3 & 138,528 & 116,005 & 0 \\
\cline { 2 - 5 } & Scenario4 & 138,529 & 116,006 & 0 \\
\hline
\end{tabular}

\section{CONCLUSIONS}

In this paper, performance analysis of three basic types of physical network topologies (bus, star and ring) using different number of connected nodes has been done using OPNET simulator. Four parameters (Delay(sec.) for complete network, Load (bits/sec.), 
Traffic Received(bits/sec.) and Number of Collision) for server node) are used to measure and analyze the performance of these topologies. Results indicate that delay for star topology increased when the number of connected nodes increased also and vice versa, while the delay in bus and ring topologies changed randomly and doesn't based on the number of connected nodes. Simulation results of three topologies show that load and traffic received of a server increased by increasing the number of connected nodes but in bus topology is more effected than two other topologies for the same number of connected nodes and especially when the number of nodes is more than 15. The number of collision for bus topology is directly proportional with the number of connected node whereas its equal to zero for star and ring topology .As a result, OPNET simulator can be used to find out the maximum number of nodes that should be connected to a network using any topology that can be withstand by a server. So we can find out the breakdown of a server before the practical implementation of a network.

\section{References}

1.Harmeet S., Sukhjeet S. and Rahul M., Modeling, Evaluation and Analysis of Ring Topology for Computer Applications Using Simulation, International Journal of Computer Science and Mobile Computing (IJCSMC), ISSN 2320-088X, 2 (1) ( 2013), 1.10.

2. Bobbinpreet K., OPNET based Analysis of Star Topology in Small Internetworks, International Journal of Electronics and Data Communication, 1 (1) (2012),
15.17.

3.Santanu S. and Pinaki P. A., A Study And Analysis on Computer Network Topology For Data Communication, International Journal of Emerging Technology and Advanced Engineering, ISSN 2250.2459, 3 (1) (2013), 522.525.

4.Mridula A., Rupesh D., Nitin J. and Deepak A., Comparative Analysis of Different Topologies based on Network.on.Chip Architectures, International Journal of Electronics and Communication Engineering, ISSN 0974.2166, 6 (1) (2013), 29.40.

5.Sushruta M., Lamboder J. and Aarti P., Networking Devices and Topologies : A Succinct Study, International Journal of Advanced Research in Computer Science and Software Engineering, ISSN : 2277.128X, 2 (11) (2012), 347.357.

6. Huang M. and Bode, B., A Performance Comparison of Tree and Ring Topologies in Distributed Systems, Parallel and Distributed Processing Symposium, Proceedings of 19th IEEE International Conference, ( 2005).

7.Pankaj R. and Dilpreet K., Optimizing Performance of Token Ring for Balanced and Unbalanced Load Using OPNET, International Journal of Scientific and Engineering Research, ISSN 2229.5518, 2 (6) 2011, 1.4.

8.IT Guru Academic Edition, OPNET Technologies, 2007. Retrieved on April 5, 2007 from http ://www.opnet.com/services/university/itguru_acade mic_edition.html 